\title{
Experimental studies on void detection in concrete-filled steel tubes using ultrasound
}

Wei Dong ${ }^{1}$, Zhimin $\mathrm{Wu}^{2, \star}$, Xiangming $\mathrm{Zhou}^{3}$, Yongjie Tan ${ }^{4}$,

${ }^{1}$ Associate Professor, State Key Laboratory of Coastal and Offshore Engineering, Dalian University of Technology \& Ocean Engineering Joint Research Center of DUT-UWA, Dalian 116024, P. R. China. E-mail: dongwei@dlut.edu.cn

${ }^{2}$ Professor, State Key Laboratory of Coastal and Offshore Engineering, Dalian University of Technology, Dalian 116024, P. R. China

( ${ }^{\star}$ Corresponding author). E-mail: wuzhimin@dlut.edu.cn

${ }^{3}$ Reader in Civil Engineering Design, Department of Mechanical, Aerospace and Civil Engineering, Brunel University London, Uxbridge, Middlesex UB8 3PH, United Kingdom \& Haitian Visiting Professor, State Key Laboratory of Coastal and Offshore Engineering, Dalian University of Technology, Dalian 116024, P. R. China. E-mail: xiangming.zhou@brunel.ac.uk ${ }^{4}$ Master student, State Key Laboratory of Coastal and Offshore Engineering, Dalian University of Technology, Dalian 116024, P. R. China. E-mail:yjtang@163.com

\section{ABSTRACT}

Due to shrinkage and/or inadequate compaction during concreting, voids may develop in a concrete-filled steel tube (CFST) between the concrete core and outer steel tube, which reduce the confinement effect of the steel tube on the concrete core, and further, decrease the load-carrying capacity and ductility of a CFST. In this study, an ultrasonic technique is utilized for quantifying voids in CFSTs by analyzing the ultrasound travel time in them. Four 
potential travel paths are identified in CFSTs with/without pre-set voids. By making a comparison of the experimental and theoretical ultrasound travel time, the actual ultrasound travel path is determined in CFSTs. Further, by analyzing the matrix of ultrasound travel time obtained from experiment, a novel method is proposed to generate the chromatogram of the distribution of ultrasound travel time, which is utilized to quantify the voids in a CFST. The chromatogram intuitively shows the position and geometry of the voids in CFSTs and is in reasonable agreement with the pre-set voids. This study, therefore, establishes a new method for quantifying voids in a CFST through the ultrasonic technique.

Keywords: Concrete-filled steel tube; void area; ultrasonic testing method; propagation path; chromatogram; de-bonding

\section{Introduction}

In the pursuit of good structural performance, low costs and/or a large floor space in modern structures, concrete-filled steel tubes (CFSTs) have been widely adopted as structural elements, such as truss elements in arch bridges and columns in high-rise buildings. In a CFST, the outer steel tube provides the lateral confinement to the concrete core so that the concrete compressive strength can be significantly enhanced. Meanwhile, local buckling of the steel tube can be restrained by filling it with concrete, resulting in a CFST's load carrying capacity to be greater than the sum of the individual component load carrying capacities i.e. steel tube and concrete core column [1]. To achieve the expected confinement effects with loading being transferred between the two materials, it is necessary to ensure excellent bonding between the concrete core and the steel tube. However, de-bonding between 
concrete and steel is almost inevitable in a CFST, which can be classified into two categories,

47 i.e. shrinkage voids and near-wall cavity voids caused by deviation in deformation between steel and concrete [2]. The defects in a CFST can reduce the confinement effect of the steel tube on concrete, consequently decreasing its load-carrying capacity and ductility. Therefore, it is significant to quantify the internal defects in a CFST to assess its structural performance using the nondestructive testing (NDT) methods. community, such as ultrasonic testing [3], acoustic emission [2], infrared thermography [4] and the impact-echo method [5]. Among them, the ultrasonic testing method has become one of the most popular NDT techniques due to its versatility and convenient mode of operation. It has been verified as a promising technique for the evaluation of crack propagation in concrete [6, 7], delamination of concrete bridge decks [8], defects inside plain and reinforced concrete [9], corrosion of steel reinforcement [10] and local yielding of steel structures [11]. However, it is still very challenging to use the traditional ultrasonic testing method to detect the de-bonding in composite elements in civil engineering structures, e.g. fiber reinforced plastic (FRP) confined concrete and CFSTs. Although the ultrasonic tomography can detect voids in concrete, the results are usually affected by the sizes and shapes of coarse aggregates [12]. Particularly, it is difficult for ultrasonic waves to penetrate deep into a highly attenuative material, e.g. epoxy resins used in the matrix of composite materials. In response to this, Feng et al. [13] developed an electromagnetic imaging technology to detect the de-bonding between FRP and concrete. However, the electromagnetic ultrasonic waves failed to penetrate the steel tube. Therefore, it cannot be 
used for assessing CFSTs. Meanwhile, some researchers tried to utilize ultrasound to evaluate defects in CFSTs based on the difference in velocities travelled by ultrasound in steel and concrete [14, 15], respectively. Their results showed that concrete quality in a CFST can be qualitatively classified based approximately on the difference in ultrasonic velocity picked up from experiment. However, it is almost impossible to determine the positions and geometries of voids in CFSTs using their method. Therefore, when quantitative detection of de-bonding (i.e. determination of both the positions and geometries of voids) in CFSTs is a matter of concern, the extent of application and effectiveness of ultrasonic technology remains as an under-researched topic. Recently, a piezoelectric (PZT) ceramic transducer-based method [16] has been widely used in the detection of de-bonding in composite reinforced concrete [17], steel reinforced concrete [18, 19] and CFSTs [20, 21]. However, it is necessary to embed these sensors into concrete during its casting, which limits its applications on existing structures [22]. Therefore, the development and implementation of NDT technology for convenient and direct detection of de-bonding in CFSTs is still a challenge in civil engineering field.

In response to this problem, this paper aims at developing a de-bonding detection method for CFSTs using the traditional ultrasonic technology. The previously mentioned ultrasound methods identified the de-bonding based on the variations in ultrasound velocity, amplitude, and frequency, however, the proposed method in this study will employ only the ultrasound travel time to quantify the two types of de-bonding. By comparing the ultrasound travel time obtained from experiment and theoretical analyses, the ultrasound travel paths in CFSTs with the two categories of de-bonding voids can be obtained. Moreover, by analyzing the 
ultrasound travel time obtained from experiment at a number of testing points in a CFST, a matrix of ultrasound travel time can be derived. Further, the chromatogram of the distribution of ultrasound travel time can be constructed, which can intuitively reflect the outline of voids, consequently enabling quantitative evaluation of defects in a CFST. It is expected that the method proposed in this study can provide new insight by using ultrasound travel time, as the only required input data, for detecting the position and geometry of voids in a CFST. This would characterize ultrasonic technology as a simple, convenient and effective technique with an inherent low cost for detection of voids in CFSTs.

\section{Ultrasound travel path}

The two categories of de-bonding in CFSTs depend on the origins of formation [2]. One is caused by concrete shrinkage, resulting in circumferential voids between the concrete core and the outer steel tube. Its characteristic is that the thickness and length of the de-bonding gap are small, but the scope along the circumference is large. The other category of de-bonding is caused by the poor compaction during concrete casting, resulting in voids forming near the inner wall of a steel tube. Its characteristic is that the length of the de-bonding area along the axial direction of a CFST is large, but the scope along the circumference is small. According to Fermat's principle, the ultrasonic wave would be transmitted along the path of least travel time. Since the ultrasound velocity is $340 \mathrm{~m} / \mathrm{s}$ in air and $4000-6000 \mathrm{~m} / \mathrm{s}$ in concrete, the ultrasonic wave may bypass the void area and transmit along the path of least travel time. Therefore, the ultrasound travel time $\tau$ will increase if there are defects existing in a CFST. Meanwhile, the path of the ultrasonic wave is complex 
112 due to the effect of the steel tube wall. Therefore, the path should be determined in advance 113 to quantify voids in a CFST.

114 When the ultrasonic actuator and receiver are symmetrically positioned on the points $A$ and 115 A' (see Fig.1) along the radian direction of the circular cross-section of a CFST, there are 116 four potential paths that the ultrasonic wave can transmit in a CFST: (1) penetrate the wall of 117 the steel tube and the concrete core of a CFST with no void, i.e. straight from Points A to A' 118 as shown in Figure. 1 (a); (2) bypass the void area and transmit along the wall of the steel 119 tube from Points $A$ to $B$, then travel straightly through the concrete, i.e. from Points $B$ to $A^{\prime}$ as 120 shown in Figure. 1 (b); (3) penetrate the void area and transmit straight through concrete, i.e. 121 from Points A to A' as shown in Figure. 1(c); and finally (4) transmit along the wall of the steel 122 tube only, i.e. from Points A to A' through Point B as shown in Figure. 1(d) without through 123 concrete.

124 In a well-compacted CFST, the ultrasonic wave will travel following Path 1 as shown in 125 Figure.1 (a). The corresponding ultrasound travel time $\tau_{1}$ can be calculated using Eq. (1).

$$
\tau_{1}=\frac{2 t}{v_{s}}+\frac{D-2 t}{v_{c}}
$$

127 where, $t$ is the thickness of the steel tube wall, $D$ is the outer diameter of the steel tube, $v_{s}$ is 128 the ultrasound velocity in steel, and $v_{c}$ is the ultrasound velocity in concrete.

129 In a CFST with a void, the potential ultrasound travel paths are Paths 2, 3 and 4 as shown in 130 Fig. 1, and have the corresponding ultrasound travel time $\tau_{2}, \tau_{3}$ and $\tau_{4}$ which can be 131 calculated using Eqs. (2), (3) and (4), respectively, as following: 


$$
\begin{gathered}
\tau_{3}=\frac{2 t}{v_{s}}+\frac{d}{v_{a}}+\frac{D-2 t-d}{v_{c}} \\
\tau_{4}=\frac{2 t+0.5 \cdot \pi(D-2 t)}{v_{s}}
\end{gathered}
$$

where, $L_{A B}$ is the arc length between points $A$ and $B$ in Fig. 1 (b); $L_{B A^{\prime}}$ is the distance between points B and $\mathrm{A}^{\prime} ; v_{a}$ is the ultrasound velocity in air; and $d$ is the thickness of the void area along the travel path. Such a method will ensure that the actual travel path is determined by comparing the travel times of the three potential travel paths. The travel path with the shortest travel time will be the actual travel path of the ultrasonic wave.

\section{Experimental program}

\subsection{Preparation of CFST specimens}

There are 6 CFST circular columns tested in this study, with an outer diameter of $219 \mathrm{~mm}$, a height of $500 \mathrm{~mm}$, and a steel tube wall thicknesses of $5.3 \mathrm{~mm}$. The CFST is made of grade Q235 steel, and grade C50 concrete. The 6 specimens were divided into two groups. 3 specimens, denoted as NT-1, NT-2 and NT-3, were used to demonstrate the detection of the first-category voids, i.e. defects caused by concrete shrinkage while the other 3 specimens, denoted as NT-4, NT-5 and NT-6, were used to demonstrate the detection of the second-category voids, i.e. defects caused by poor compaction in construction. Taking the 150 specimens NT-2 and NT-5 as an example, Fig. 2 shows the two categories of voids in CFSTs.

152 In the case of the first-category void, the thicknesses of spacer $d$ were 3.0, 4.5 and $7.0 \mathrm{~mm}$ 153 for specimens NT-1, -2 , and -3 , respectively. The geometries and position of the spacer are 
sketched in Fig. 3. Before casting concrete, the spacers were closely attached on the inner surface of the wall of the steel tube. They were removed right after the initial setting of concrete. By such measures, pre-set voids with various thicknesses and arc lengths were obtained, which were distributed along the axial direction of the CFST specimens in a ladder-like manner. The cross-sections of the first-category void at the heights of 150 to 250 mm, 250 to $350 \mathrm{~mm}$ and 350 to $400 \mathrm{~mm}$ are shown in Fig. 4 (a), (b) and (c), respectively. It can be seen that void area increases with height.

In the case of the second-category void, the thicknesses of spacer $d$ were 10, 30 and $50 \mathrm{~mm}$ for specimens NT-4, -5 , and -6 , respectively. The geometries and position of the spacer are category void are shown in Fig. 6 (a), (b) and (c), respectively.

\subsection{Distribution of testing points}

The ultrasonic generator used for this study was a TICO-series ultrasonic generator produced by Proceq in Switzerland. The ultrasound used for this study had a frequency of 54 $\mathrm{kHz}$. Each CFST column was divided into 10 segments of equal length along its axial direction. The testing sections were set from 100 to $450 \mathrm{~mm}$, with $50 \mathrm{~mm}$ interval, above the column footing, denoted as Sections $\mathrm{A}$ to $\mathrm{H}$. At each section, 12 testing couples were set at $15^{\circ}$ interval along its outer circumference. In total 12 pairs of the ultrasonic actuators and receivers were symmetrically mounted on the outer surface of the steel tube of each CFST 
column along its circumferential direction, such as 1-1', 2-2' etc. as illustrated in Figs. 4 and 6. Therefore, in total 96 testing points were set for each CFST specimen as shown in Fig. 7.

\section{Results and discussions}

\subsection{Ultrasound travel in compact CFSTs}

181 To get a stable ultrasound travel time, all tests were carried out at the concrete age of 60 days. Meanwhile, 100-mm concrete cubes were cast in parallel with the CFST specimens to test the ultrasound velocity in concrete also at the age of 60 days. The steel tubes prepared for the experiment were used to test the ultrasound velocity in steel before filling with concrete. The average ultrasound velocities measured in concrete and steel were 4880 and $5620 \mathrm{~m} / \mathrm{s}$, respectively, in this study. To measure the ultrasound travel time in a CFST with well-compacted concrete, one testing section from each of the NT-3, $-4,-5$ and -6 specimens was selected. These testing sections are denoted as NT-3-B, NT-4-A, NT-5-F and NT6-C, respectively. For instance, NT-4-A denotes section A, with the height of $50 \mathrm{~mm}$ above the footing, of specimen NT-4. The measured ultrasound travel times corresponding to the 12 couples of testing points at each section are listed in Table 1. According to Eq. (1), the theoretical value of $\tau_{1}$ should be $44.6 \mu$ s in this study, which is in agreement with the experimental results, which ranged between 43.7 and $48.6 \mu \mathrm{s}$, as shown in Table 1. CFST specimens is Path 1 as illustrated in Fig. 1 (a). 
With respect to the first-category voids in CFSTs, experimental results of specimen NT-1 and NT-3 are listed in Table 2, in which six testing sections, i.e., sections $\mathrm{C}$ to $\mathrm{H}$ of each specimen were investigated to measure the ultrasound travel time. It can be seen from experimental results that there are some significantly larger values of travel time measured in certain testing points compared with neighbouring testing points, which are highlighted in italic and underlined in Table 2. The range of the highlighted data is approximately ladder-like, which has reasonable agreement with the pre-set void distribution in the corresponding CFST specimen, suggesting the results are reliable.

If the ultrasound travels along Path 4 , its travel time should be determined by the semi-perimeter of the CFST specimen. Then, the travel time measured by each couple of testing points along the circumference of a CFST specimen should be the same, which is $61.2 \mu$ s according to Eq. (4). Similarly, if the ultrasonic wave travels along Path 3, its travel time should be related to the thickness of the void when the void exists between a couple of testing points. Then, the travel time measured by each couple of testing points should be 52.8 and $63.7 \mu \mathrm{s}$, according to Eqs. (3), with respect to specimens NT-1 and NT-3, respectively. However, by comparing with the experimental results in Table 2, the theoretical travel time along Paths 3 and 4 shows distinct differences. Therefore, it can be confirmed that the ultrasonic waves are not transmitted along Path 3 or 4 for the first-category void in a CFST. If the ultrasonic waves transmit along Path 2, the theoretical travel time can be calculated using Eq. (2), which is listed in Table 3. It should be noted that according to Eq. (2), the void has no effect on the calculated results as the ultrasound bypasses the void, therefore, the theoretical travel time should be the same for specimens NT-1. -2 and -3 . 
Comparing the highlighted data in Tables 2 and 3, it can be seen that both the distribution and values of the highlighted data in the two tables are in reasonable agreement with each other, indicating that the ultrasound should transmit along Path 2 in a CFST with the 223 first-category voids.

\subsection{Ultrasound travel in CFSTs with the second-category voids}

226 The same analysis method is adopted for the investigation of ultrasoud transmission in 227 CFST specimens with the second-category voids. In this case, two testing sections, H and G, were investigated for each specimen and the measured ultrasound travel time in specimen NT-4, -5 and -6 are listed in Table 4 . It can be seen that the distribution of enlarged values of travel time, as highlighted in italic and underscored in this table, reflects the positions of voids in the three specimens (see Fig. 6). If the ultrasonic wave travels along Paths 4, a uniform travel time, i.e. $61.2 \mu$ s, should be obtained from experiment as per Eq. (4). Meanwhile, if the ultrasound travels along Path 3, the theoretical maximum travel time in specimen NT-4, -5 and -6 should be $72.0 \mu \mathrm{s}, 126.7 \mu \mathrm{s}$ and $181.4 \mu \mathrm{s}$, respectively, based on

Eq. (3). Therefore, it can be confirmed that the ultrasound did not travel along Path 3 or 4 in 237 the experimental ones listed in Table. 4. If the ultrasound travels along Path 2, the theoretical 238 travel time can be calculated using Eq. (2), which are listed in Table 5. Compared with the highlighted data in Table 4, it can be seen that both the distribution and values of the highlighted data in the two tables have a reasonable agreement, which indicates that the ultrasound does travel along Path 2 in the CFST specimens with the second-category voids. 
242 It should be noted that, in general, the ultrasound travel time obtained from experiment is a 243 bit greater than the corresponding theoretical ones for CFST specimens for both categories 244 of voids. Particularly, the experimental data becomes increasingly larger when the testing 245 points are close to the edge of void areas, such as the testing points NT-5-H-9 and NT-1-D-8. 246 By examining these areas, it can be found that, usually, there exists an imperfect contact 247 between the steel tube and concrete, e.g. loose and porous concrete filled inside. However, 248 the increment in the ultrasound travel time does not affect the identification of the voids. On 249 the contrary, it makes the qualitative detection of void edges in a CFST much easier.

\section{Chromatogram of ultrasound travel time distribution}

252 Although the void areas can be approximately detected based on the analysis of ultrasound travel time listed in Tables 2 and 4, improvement is needed for quantifying voids, for example, the judgment on the void edges is inaccurate and not intuitive. It is inconvenient to compare the data one by one, which may result in the omission and mistake of the determination of the voids. Therefore, the following research aims at imaging the voids in the CFSTs based on the ultrasound travel time obtained in experiment. In summary, the following steps are included in developing the images of voids in CFSTs:

1. Generate grids based on the testing points, which are the nodes of the grids generated. Taking specimen NT-1 as an example, there is ultrasound travel time from $8 \times 12$ testing points so that the half-cylinder can be on average divided into 8 $\times 12$ grids, in which 8 grids along the axial direction and 12 grids along the circumferential direction. 
2. Form the matrix of ultrasound travel time. By assigning the ultrasound travel time to the corresponding nodes, the matrix can be obtained, in which the elements are the ultrasound travel time.

3. Densify the grids. To get a more accurate void area in the derived images, the grids should be re-fined so that the smooth edge of the void can be obtained. In this study, each grid derived from the previous step is divided into $50 \times 50$ grid nodes, making the half-cylinder be divided into $400 \times 600$ grid nodes.

4. Form the densified matrix of ultrasound travel time. Through introducing the V4 interpolation method provided by MATLAB software, the ultrasound travel time at each of the $400 \times 600$ grid nodes can be obtained.

5. Draw the chromatogram of the distribution of ultrasound travel time using MATLAB based on the densified matrix obtained in step 4.

Using the abovementioned steps of analysis, the chromatogram of the CFST specimens tested in this study can be derived, and are illustrated in Fig. 8. In the case of the first-category voids, i.e., in specimens NT-1, -2 and -3 , it can be clearly seen that the void heights are $200-450 \mathrm{~mm}$ from the footing of specimens, and are in approximately a ladder-like distribution along the axial direction (see Fig. 8 (a), (b) and (c)). Meanwhile, in the case of the second-category voids, i.e., in specimens NT-4, -5 and -6 , the figures explicitly illustrate the geometries of the voids, which have a height of $350-450 \mathrm{~mm}$ from the footing of specimens and circumferential angles from $45-75^{\circ}, 60-120^{\circ}$ and $105-195^{\circ}$, respectively (see Fig. 8 (d), (e) and (f)). It should be noted that there are some areas highlighted in red in the chromatogram, which are not pre-set voids in the experiment, such as the area with 
height of $100-250 \mathrm{~mm}$ and circumferential angle of $30^{\circ}-90^{\circ}$ shown in Fig. 8(d). Through an examination of this area after testing, it was found that some of the concrete filled inside was loose and porous, increasing the ultrasound travel time. Therefore, by comparing with the pre-set voids in CFST specimens as shown in Figs. 4 and 6, the derived chromatogram shows a reasonable agreement with respect to the positions and geometries of the voids.

To study the effects of concrete age using the chromatogram, similar tests were conducted on the CFST specimens at the ages of 7,28 and 90 days with the results presented in Fig. 9. Although the color is slightly different in the chromatograms, the geometries of the detected voids are almost the same, suggesting that concrete age has little effects on the determination of voids, so that the chromatogram method proposed in this study can be employed for detecting the voids in CFSTs both under construction and in service.

In practical engineering, detecting the voids more effectively and at the lowest possible cost is of prime importance. Therefore, it is necessary to study the effects of the number of testing points on the accuracy of detection of the voids. Taking specimen NT-3 as an example, 4, 6 and 12 couples were utilized to test the ultrasound travel time, and then form the corresponding chromatograms, which are shown in Fig. 10(a), (b) and (c), respectively. When 4 couples of testing points are adopted, the obtained chromatogram of the void area is blurred and spread (see Fig. 10 (a)). The height of the void can be determined roughly, but the range along the circumference demonstrates large deviation, in which only one ladder-like distribution pattern can be found. With the increase of testing points from 4 to 6 couples, the derived chromatogram can approximately reflect the pre-set voids in the 
chromatogram has a better agreement with the experimental results (see Fig. 10 (c)). However, compared with Fig 10 (b), the improvement in Fig 10 (c) is not significant.

310 Therefore, it is concluded that 6 couples of testing points can appropriately meet the 311 requirement of void detection in practical engineering

312 It is worth pointing out that the symmetrical ultrasonic testing and the imaging methods can 313 be used only in the case that the ultrasonic wave travels along Path 2 shown in Fig. 1, i.e., $314 \tau_{1}<\tau_{2}<\tau_{4}$. The minimum central angles corresponding to the voids can be determined 315 according the criteria of $\tau_{1}<\tau_{2}$, and the maximum one can be determined according to the 316 criteria $\tau_{2}<\tau_{4}$. Through substituting Eqs (1), (2) and (4) into the equations, the scopes of the 317 central angles corresponding to the voids are listed in Table 6. It should be noted that the 318 minimum central angles are less influenced by the ultrasonic wave velocities in different concrete, so the values can be approximately determined as $12^{\circ}$. The scenario is different in 320 the case of the determination of maximum central angles. Since the ultrasonic wave 321 velocities in different concrete vary from 4000 to $5400 \mathrm{~m} / \mathrm{s}$, the corresponding maximum 322 central angle ranges from 22 to $172^{\circ}$ (see Table 6). Therefore, the minimum/maximum sizes 323 of voids that can be detectable depend on the diameter of CFST cross-section. In case of 324 smaller CFST, the minimum /maximum sizes of voids that can be detected are smaller. In contrast, the sizes are greater for a bigger diameter CFST. Taking the CFST used in this 326 study as an example, the minimum size of void that can be detected is $20.7 \mathrm{~mm}$. 327 Considering that the concrete strength grade for CFSTs should be greater than C50 for the purpose of maximizing their advantages in structures, the corresponding maximum central angle can be greater than $110^{\circ}$. Therefore, the symmetrical ultrasonic testing and imaging 
methods are effective and suitable for the detection of de-bonding voids in the CFST members.

332 Moreover, the scope of the void is determined by the actual ultrasound travel path, therefore, 333 the shape of the void will affect the measurement in certain case. For example in Fig 1(b), 334 the ultrasound travel between Points B and A' of concrete is not along a line, i.e. the void 335 enters the area surrounded by Line BA' and Arc BA'. In this case, the derived ultrasound 336 travel time will get longer so that the predicted void area will be larger than the actual one, 337 resulting in an over-estimation on the void size.

\section{Conclusions}

340 In this study, the symmetrical ultrasonic testing method was introduced to detect the 341 de-bonding voids in CFSTs. By investigating the variation of ultrasound travel time in the 342 CFST specimens with different categories of pre-set voids, the ultrasound travel path was 343 determined. Based on the experimental results from various testing points, the matrix of 344 ultrasound travel time can be obtained, in which elements of rows and columns represent 345 the results in the circumferential and the axial direction, respectively. To get a more accurate 346 image of voids in the CFST members, a densified matrix can be derived by interpolation of 347 the original matrix of ultrasound travel time. Finally, the chromatogram of the distribution of 348 ultrasonic wave travel time based on the derived densified matrix can be drawn using 349 MATLAB. The effects of concrete age and quantity of testing points on the chromatogram were analyzed, and the scope of the symmetrical ultrasonic testing and imaging methods in 351 the detection of de-bonding voids in CFST members was discussed. Based on experimental 
and theoretical studies, the following conclusions can be drawn:

(1) For the CFST members without a void, the ultrasonic waves penetrate the steel tube wall and concrete and are transmitted in a straight line. In the case of CFST members with a de-bonding void, the ultrasonic waves bypass the void area along the wall of the steel tube and then travel through the concrete along a straight line.

(2) Based on the matrix of ultrasonic wave travel time obtained in experiment, the chromatogram is derived, which can effectively and intuitively reflect the location and geometry of de-bonding void areas in CFST members.

(3) Concrete ages have little effect on the determination of voids in CFSTs using the chromatogram so that the method proposed in this study can be used to evaluate the de-bonding in the CFST members under construction and in service.

(4) To reflect the position and geometry of the void area, at least 6 couples of testing points must be arranged equally along the circumferential direction in a testing section. When the number of testing points decreases to 4 couples, the obtained chromatogram may be distorted resulting in an incorrect judgment on the void geometry.

\section{Acknowledgement}

The financial support of the National Natural Science Foundation of China under the grants of NSFC 51478084, 51421064, and 51478083, partial finance support from the UK Royal Academy of Engineering through the Distinguished Visiting Fellow scheme under the grant DVF1617_5_21is gratefully acknowledged.

\section{References}


375 [1] Shanmugam NE, Lakshmi B. State of the art report on steel-concrete composite columns. 376 J Constr Steel Res. 2001;57:1041-80.

377 [2] Xue J-Q, Briseghella B, Chen B-C. Effects of debonding on circular CFST stub columns. 378 J Constr Steel Res. 2012;69:64-76.

379 [3] Iyer S, Sinha SK, Tittmann BR, Pedrick MK. Ultrasonic signal processing methods for 380 detection of defects in concrete pipes. Automat Constr. 2012;22:135-48.

381 [4] Clark MR, McCann DM, Forde MC. Application of infrared thermography to the 382 non-destructive testing of concrete and masonry bridges. Ndt\&e Int. 2003;36:265-75.

383 [5] Hola J, Sadowski L, Schabowicz K. Nondestructive identification of delaminations in 384 concrete floor toppings with acoustic methods. Automat Constr. 2011;20:799-807.

[6] Wolf J, Pirskawetz S, Zang A. Detection of crack propagation in concrete with embedded ultrasonic sensors. Eng Fract Mech. 2015;146:161-71.

[7] Godinho L, Dias-da-Costa D, Areias P, Júlio E, Soares Jr D. Numerical study towards the use of a SH wave ultrasonic-based strategy for crack detection in concrete structures. Eng Struct. 2013;49:782-91.

[8] Shokouhi P, Wolf J, Wiggenhauser H. Detection of delamination in concrete bridge decks by joint amplitude and phase analysis of ultrasonic array measurements. J Bridge Eng. 2014;19:11.

[9] Wang C-Y, Liao S-T, Tong J-H, Chiu C-L. Numerical and experimental study on multi-directional SAFT to detect defects inside plain or reinforced concrete. Constr Build Mater. 2015;76:351-9.

[10] Di Benedetti M, Loreto G, Matta F, Nanni A. Acoustic Emission Monitoring of Reinforced 
Concrete under Accelerated Corrosion. J Mater Civil Eng. 2012;25:1022-9.

[11] Bingol Y, Okeil A. Ultrasonic signal characteristics for nondestructive-yield detection in steel structures. J Mater Civil Eng. 2014;27:04014271.

[12] Buyukozturk O. Imaging of concrete structures. Ndt\&e Int. 1998;31:233-43.

[13] Feng M, De Flaviis F, Kim Y. Use of microwaves for damage detection of fiber reinforced polymer-wrapped concrete structures. J Eng Mech. 2002;128:172-83.

[14] Zhang H, Yu Q, Lu Y. Application study of quality testing of arch-rib concrete of concrete filled steel tube arch bridge by ultrasonic transmission method China Civil Eng J. 2004;37:50-3.

[15] Liu Y, Zhang Y, Zou Z. Study of ultrasonic detection and evaluation for the deficiency of concrete-filled steel tube arch bridge. Journal of Northern Jiaotong University. 2004;28:54-7. [16] Park G, Cudney HH, Inman DJ. Feasibility of using impedance-based damage assessment for pipeline structures. Earthquake Eng Struc. 2001;30:1463-74.

[17] Saafi M, Sayyah T. Health monitoring of concrete structures strengthened with advanced composite materials using piezoelectric transducers. Compos Part B-eng. $2001 ; 32: 333-42$.

[18] Wu F, Chang FK. Debond detection using embedded piezoelectric elements for reinforced concrete structures - Part II: Analysis and algorithm. Struct Health Monit. 2006;5:17-28.

[19] Wu F, Chang FK. Debond detection using embedded piezoelectric elements in reinforced concrete structures - Part I: Experiment. Struct Health Monit. 2006;5:5-15.

[20] Bin X, Bing L, Gangbing S. Active debonding detection for large rectangular CFSTs 
419 based on wavelet packet energy spectrum with piezoceramics. J Struct Eng. $420 \quad 2013 ; 139: 1435-43$.

421 [21] Xu B, Zhang T, Song G, Gu H. Active interface debonding detection of a concrete-filled 422 steel tube with piezoelectric technologies using wavelet packet analysis. Mech Syst Signal 423 Pr. 2013;36:7-17.

424 [22] Chang PC, Liu SC. Recent research in nondestructive evaluation of civil infrastructures. 425 J Mater Civil Eng. 2003;15:298-304. 


\section{Captions of figures}

442 Fig. 1. Four potential paths of ultrasound transmission: (a); (b); (c); and (d)

443 Fig. 2. Two categories of pre-set voids in CFST specimens: (a) First-category void;

444 (b) Second-category void

445 Fig. 3. Spacer geometry and void position in CFST specimens with the pre-set first-category 446 void

447 Fig. 4. Cross sections of a CFST specimen at various heights with the pre-set first-category 448 void: (a) Height of 150-250 mm; (b) Height of 250-350 mm; and (c) Height of 350-400 mm 449 Fig. 5. Spacer geometry and void position in specimens with the pre-set second-category 450 void

451 Fig. 6. Cross sections of CFST specimens with the pre-set second-category void with 452 different void thickness: (a) $d=10 \mathrm{~mm}$; (b) $d=30 \mathrm{~mm}$; and (c) $d=50 \mathrm{~mm}$

453 Fig. 7. Distribution of testing points along axial and circumferential directions of a CFST 454 specimen (unit: $\mathrm{mm}$ )

455 Fig.8. Chromatogram of the distribution of ultrasound travel time: (a) NT-1; (b) NT-2; (c) 456 NT-3; (d) NT-4; (e) NT-5; and (f) NT-6

457 Fig. 9. Chromatograms of specimen NT-3 at various concrete ages: (a) 7 days; (b) 28 days; 458 and (c) 90 days

459 Fig. 10. Chromatograms of specimen NT-3 with different numbers of testing couples: (a) 4 460 couples; (b) 6 couples; and (c) 12 couples 


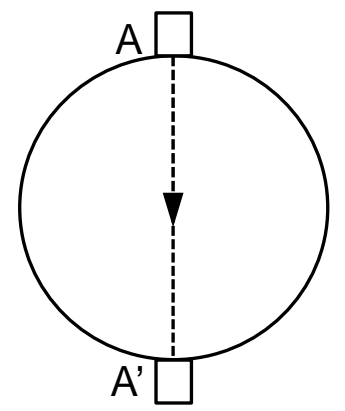

(a)

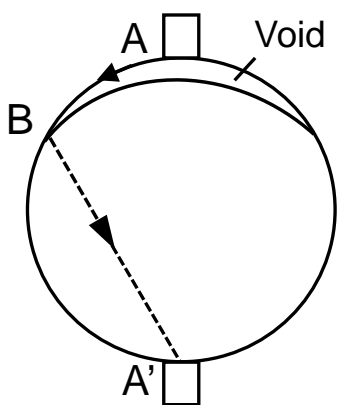

(b)

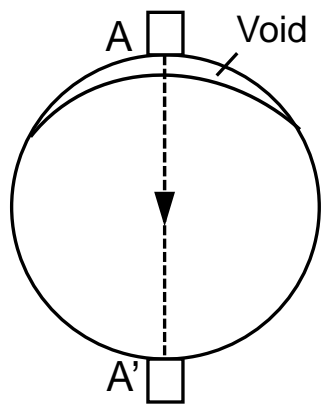

(c)

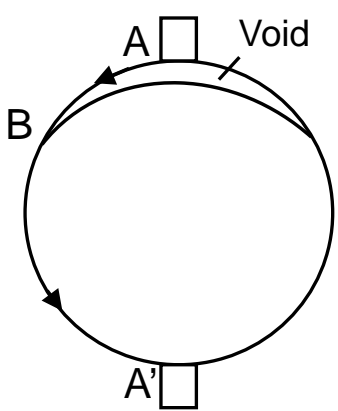

(d)

Fig. 1. Four potential paths of ultrasound transmission 


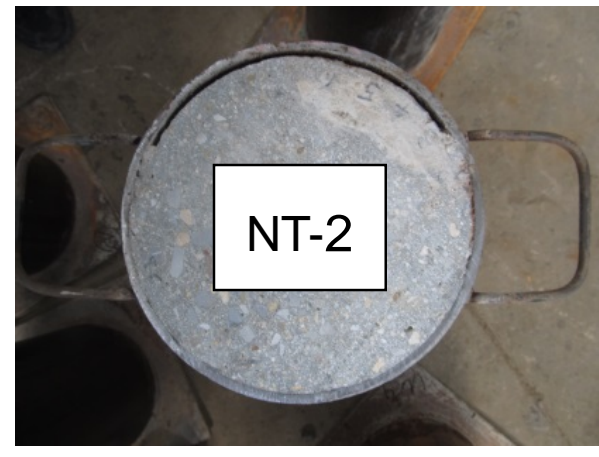

486

(a) First-category void

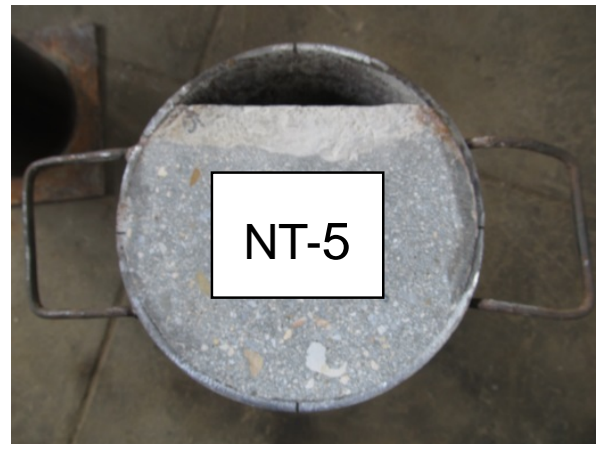

(b) Second-category void

Fig. 2. Two categories of pre-set voids in CFST specimens 


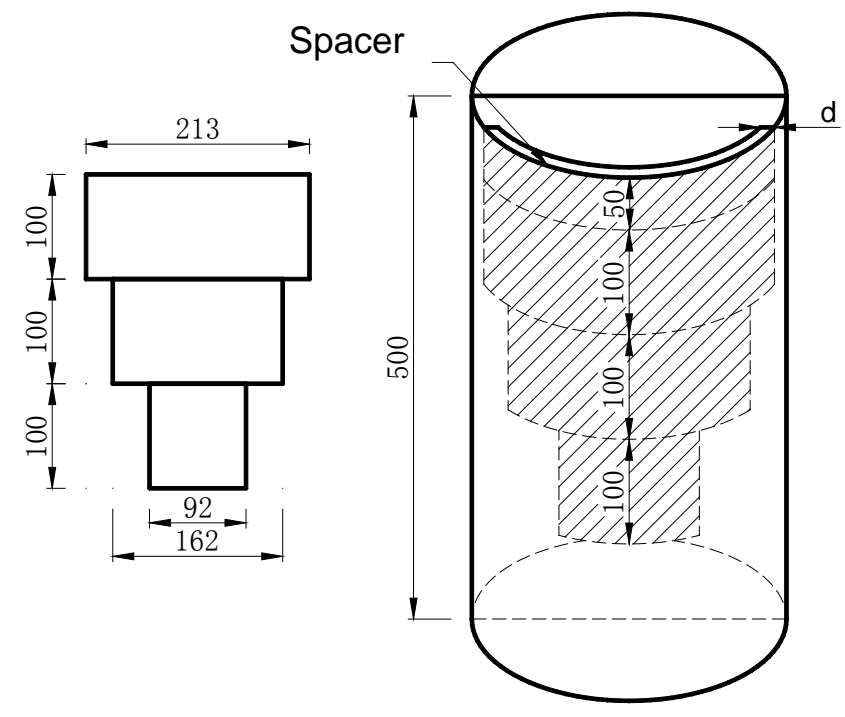

Fig. 3. Spacer geometry and void position in CFST specimens with the pre-set first-category 
520

521

522

523

524

525

526

527

528

529

530

531

532

533

534

535

536

537

538

539

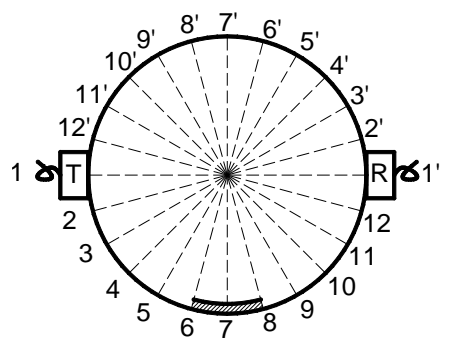

(a) Height of $150-250 \mathrm{~mm}$

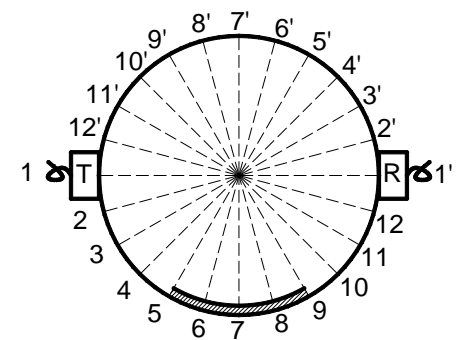

(b) Height of $250-350 \mathrm{~mm}$

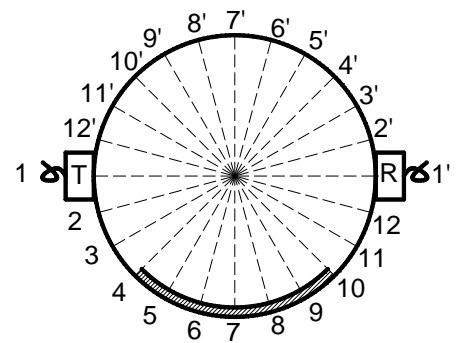

(c) Height of $350-400 \mathrm{~mm}$

Fig. 4. Cross sections of a CFST specimen at various heights with the pre-set first-category void 


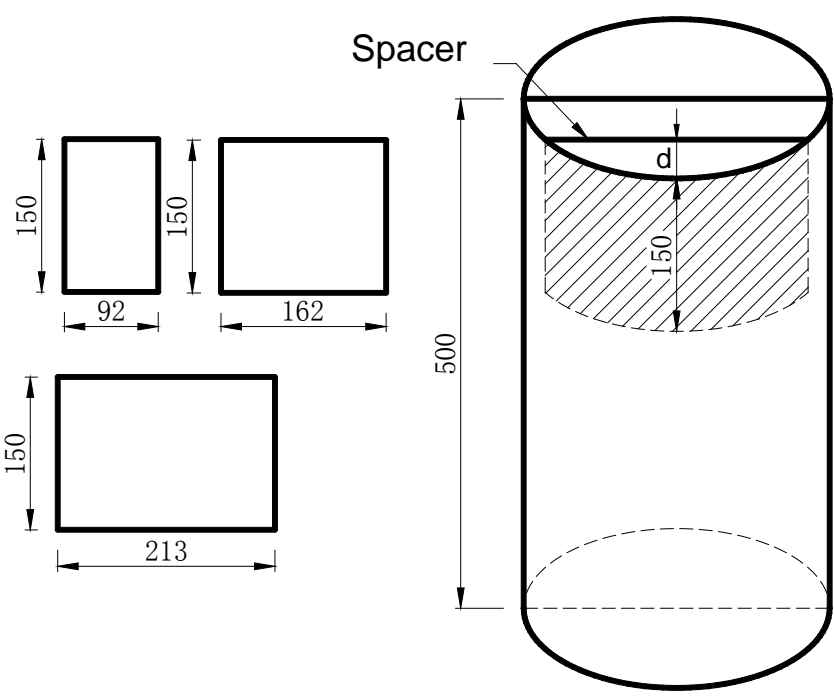

540

541

542

543

544

545

546

547

548

549

550

551

552

553

554

555

556

void

Fig. 5. Spacer geometry and void position in specimens with the pre-set second-category 


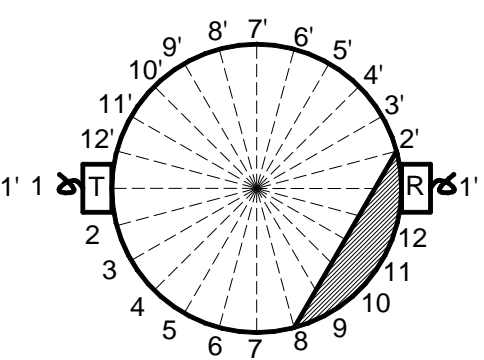

(c) $d=50 \mathrm{~mm}$

Fig. 6. Cross sections of CFST specimens with the pre-set second-category void with

different void thickness

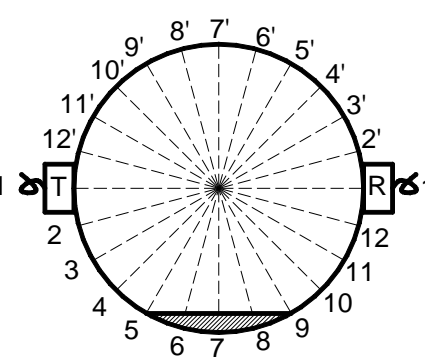

(b) $d=30 \mathrm{~mm}$ (a) $d=10 \mathrm{~mm}$

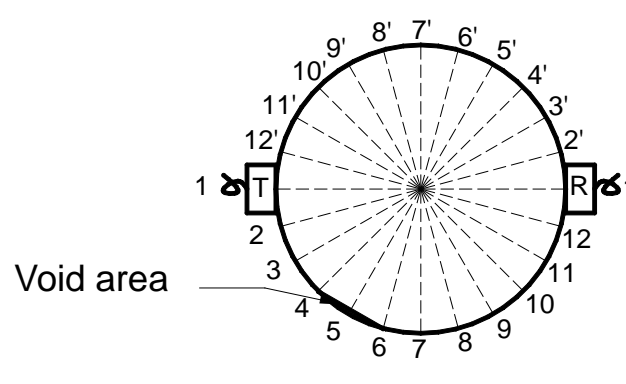


82

85

\section{6}

87

88

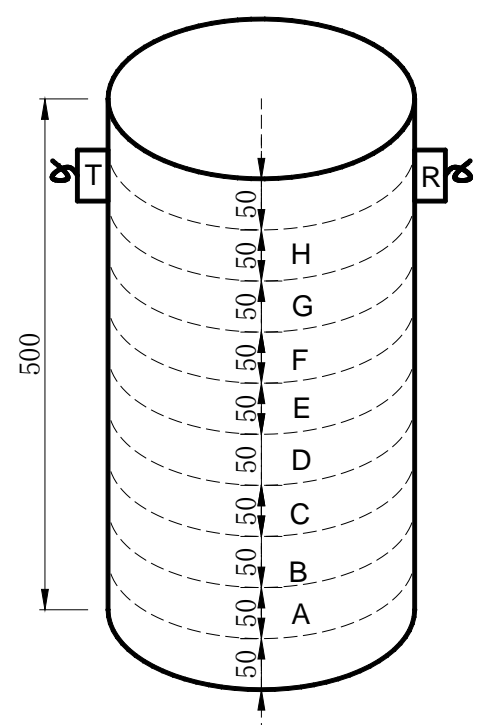

(a) axial

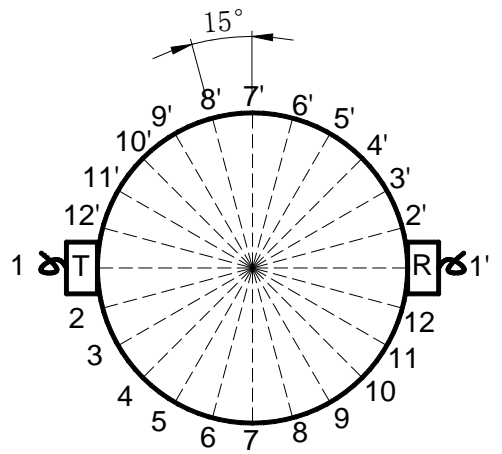

(b) circumferential

Fig. 7. Distribution of testing points along axial and circumferential directions of a CFST specimen (unit: mm)

1

3

4




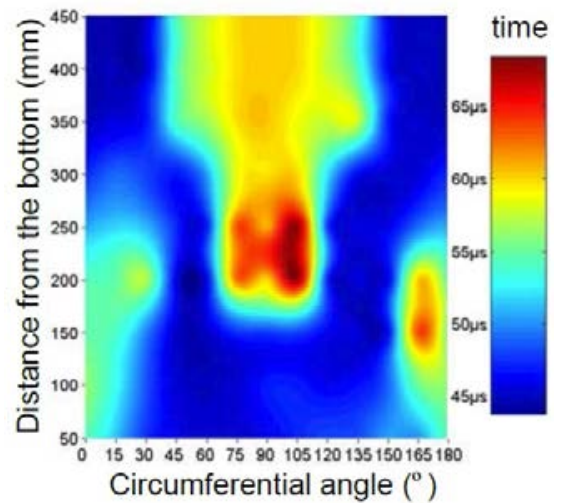

(a) NT-1

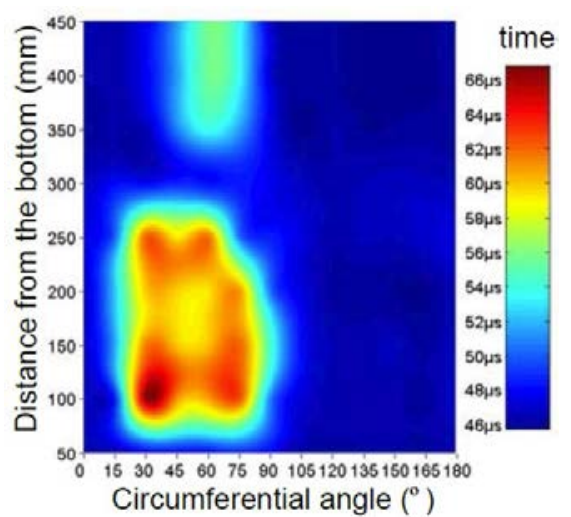

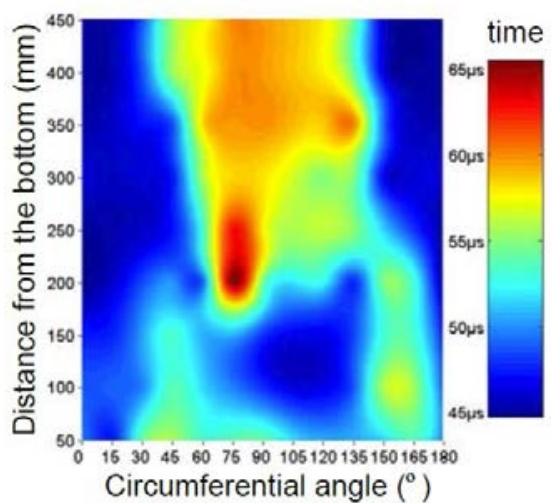

(b) NT-2

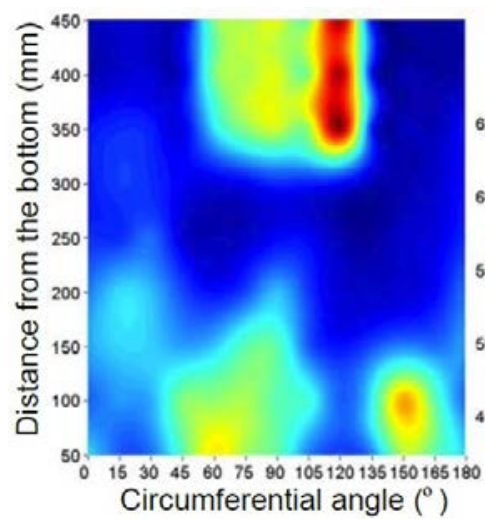

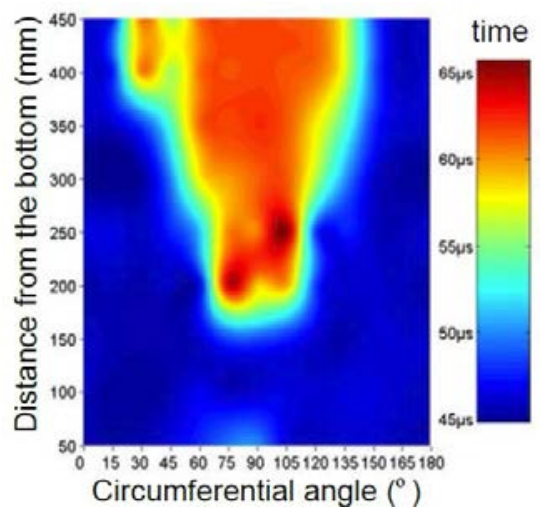

(c) NT-3

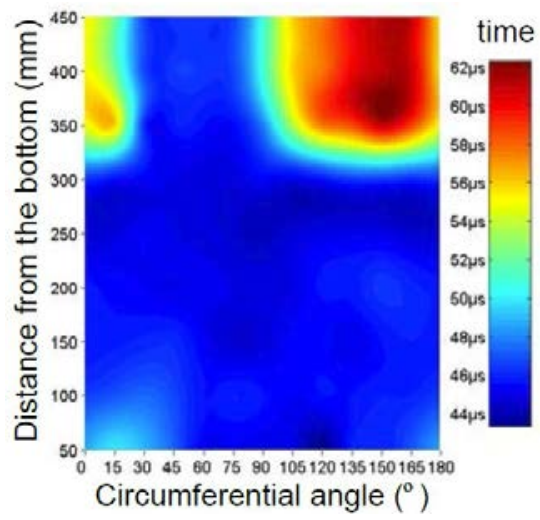

Fig.8. Chromatogram of the distribution of ultrasound travel time 


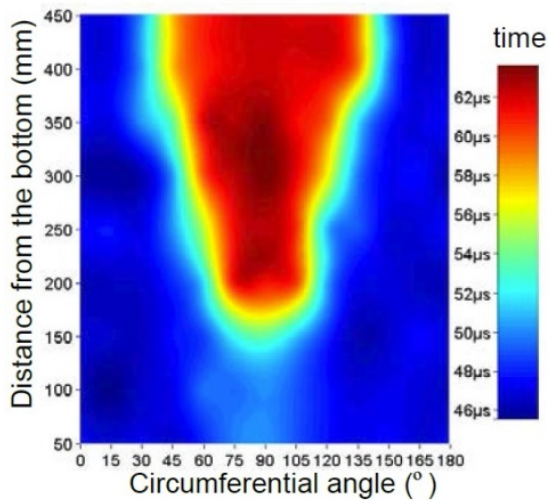

(a) 7 days

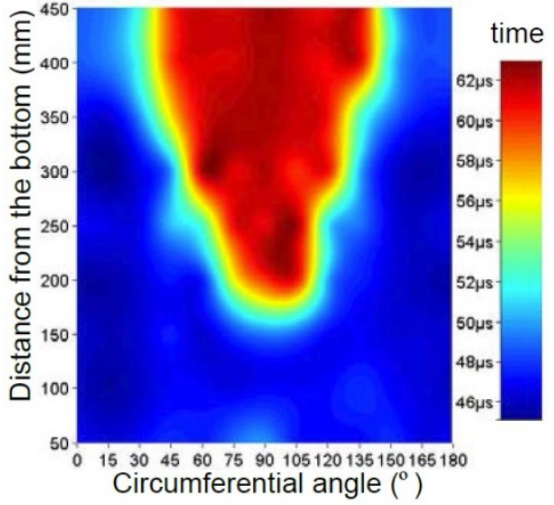

(b) 28 days

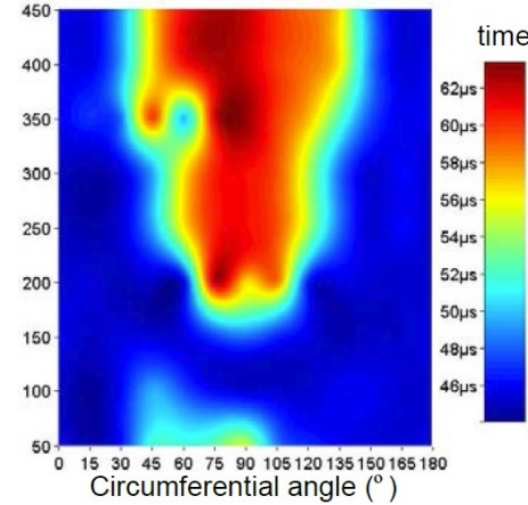

(c) 90 days

Fig. 9. Chromatograms of specimen NT-3 at various concrete ages 


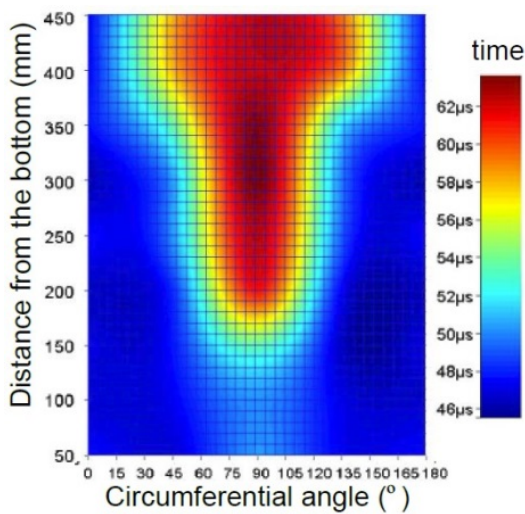

(a) 4 couples

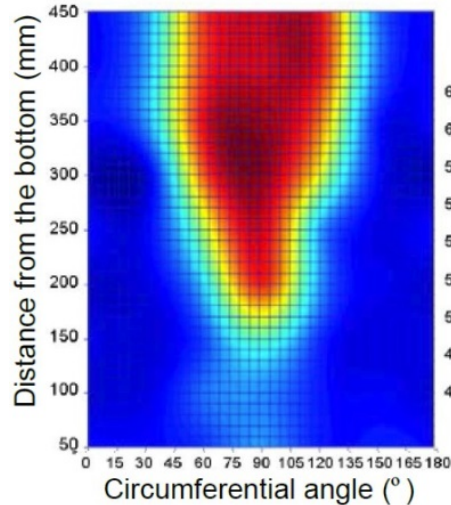

(b) 6 couples
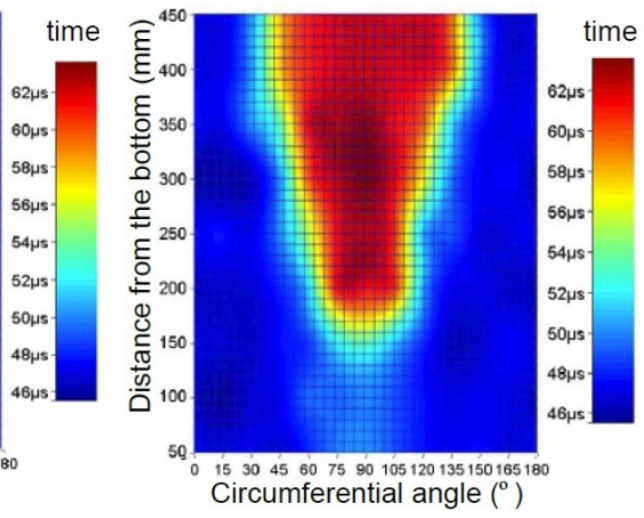

(c) 12 couples

Fig. 10. Chromatograms of specimen NT-3 with different numbers of testing couples 
645

646

647

\begin{tabular}{ccccccccccccc}
\hline Testing points & 1 & 2 & 3 & 4 & 5 & 6 & 7 & 8 & 9 & 10 & 11 & 12 \\
\hline NT-3-B & 45.7 & 45.1 & 45.1 & 45.5 & 46.8 & 45.8 & 46.2 & 46.5 & 46.1 & 46.4 & 46.4 & 46.0 \\
NT-4-A & 46.2 & 46.4 & 47.6 & 47.6 & 47.8 & 48.6 & 47.7 & 46.1 & 45.9 & 46.1 & 46.1 & 46.0 \\
NT-5-F & 47.2 & 48.2 & 47.7 & 46.5 & 46.1 & 46.6 & 47.8 & 47.4 & 46.9 & 45.0 & 44.9 & 45.5 \\
NT-6-C & 45.7 & 45.8 & 46.0 & 46.1 & 45.2 & 44.6 & 44.8 & 45.4 & 45.4 & 45.0 & 45.6 & 45.9 \\
\hline
\end{tabular}

648

649

650

651

\begin{tabular}{|c|c|c|c|c|c|c|c|c|c|c|c|c|}
\hline Testing points & 1 & 2 & 3 & 4 & 5 & 6 & 7 & 8 & 9 & 10 & 11 & 12 \\
\hline NT-1-C & 54.8 & 53.9 & 49.6 & 45.1 & 45.1 & 47.5 & 47.8 & 48.0 & 46.0 & 45.4 & 46.2 & 63.7 \\
\hline$-1-D$ & 54.3 & 54.6 & 56.9 & 45.5 & 46.0 & $\underline{62.8}$ & $\underline{60.0}$ & $\underline{67.9}$ & 46.8 & 45.8 & 45.8 & 60.2 \\
\hline$-E$ & 50.5 & 51.6 & 50.8 & 47.1 & 46.9 & 63.4 & $\underline{60.8}$ & $\underline{67.8}$ & 47.6 & 45.8 & 45.8 & 49.0 \\
\hline & 47.2 & 48.9 & 47.9 & 46.8 & $\underline{50.5}$ & $\overline{58.6}$ & $\overline{59.5}$ & $\overline{59.8}$ & $\underline{52.0}$ & 46.3 & 45.4 & 45.4 \\
\hline & & 45.6 & 46.6 & $\underline{54.1}$ & $\underline{56.5}$ & $\underline{59.7}$ & $\underline{60.3}$ & $\underline{58.6}$ & $\underline{57.6}$ & $\underline{57.8}$ & 46.7 & 45.1 \\
\hline NT-1-H & 44.3 & 44.7 & 45.0 & $\overline{54.3}$ & $\overline{57.5}$ & $\overline{59.7}$ & $\underline{60.0}$ & $\overline{59.8}$ & $\overline{57.4}$ & $\overline{54.1}$ & 45.9 & 45.1 \\
\hline & 46.0 & 45.5 & 45.4 & 45.5 & 46.1 & 48.6 & 48.7 & 47.7 & 46.2 & 45.5 & 46.2 & 46.0 \\
\hline $3-D$ & 45.2 & 45.8 & 45.3 & 46.0 & 46.8 & $\underline{64.4}$ & 58.8 & 59.7 & 48.2 & 46.3 & 46.1 & 45.8 \\
\hline & 46.3 & 46.6 & 45.8 & 47.8 & $\underline{51.3}$ & $\underline{60.3}$ & $\underline{60.4}$ & $\underline{65.5}$ & 48.2 & 47.7 & 45.9 & 45.7 \\
\hline & 44.8 & 44.8 & 45.2 & $\underline{50.0}$ & $\underline{58.1}$ & $\underline{59.8}$ & $\underline{61.1}$ & $\underline{61.3}$ & $\underline{56.2}$ & $\underline{50.4}$ & 46.1 & 45.3 \\
\hline NT-3-G & 45.9 & 46.0 & 48.4 & 53.4 & $\underline{61.1}$ & $\underline{61.6}$ & $\underline{62.3}$ & 61.3 & 58.0 & 53.8 & 47.8 & 46.2 \\
\hline NT-3-H & 46.4 & 47.7 & $\underline{60.5}$ & 55.8 & 60.9 & 61.4 & 61.8 & 61.3 & $\underline{60.8}$ & 57.2 & 48.9 & 45.7 \\
\hline
\end{tabular}

652

Table. 3 Theoretical ultrasound travel time (unit: $\mu \mathrm{s}$ ) along Path 2 in the first-category void

\begin{tabular}{|c|c|c|c|c|c|c|c|c|c|c|c|c|}
\hline Testing points & 1 & 2 & 3 & 4 & 5 & 6 & 7 & 8 & 9 & 10 & 11 & 12 \\
\hline C & 44.6 & 44.6 & 44.6 & 44.6 & 44.6 & $\underline{47.8}$ & 51.7 & 47.8 & 44.6 & 44.6 & 44.6 & 44.6 \\
\hline D & 44.6 & 44.6 & 44.6 & 44.6 & 44.6 & 47.8 & 51.7 & 47.8 & 44.6 & 44.6 & 44.6 & 44.6 \\
\hline$E$ & 44.6 & 44.6 & 44.6 & 44.6 & $\underline{49.0}$ & $\overline{52.8}$ & $\overline{55.8}$ & $\overline{52.8}$ & 49.0 & 44.6 & 44.6 & 44.6 \\
\hline$F$ & 44.6 & 44.6 & 44.6 & 44.6 & $\underline{49.0}$ & $\underline{52.8}$ & $\underline{55.8}$ & $\underline{52.8}$ & $\underline{49.0}$ & 44.6 & 44.6 & 44.6 \\
\hline G & 44.6 & 44.6 & 44.6 & 48.7 & $\underline{52.5}$ & 55.7 & 58.1 & 55.7 & $\underline{52.5}$ & 48.7 & 44.6 & 44.6 \\
\hline $\mathrm{H}$ & 44.6 & 44.6 & 44.6 & 48.7 & $\overline{52.5}$ & $\overline{55.7}$ & $\overline{58.1}$ & 55.7 & $\underline{52.5}$ & 48.7 & 44.6 & 44.6 \\
\hline
\end{tabular}

Table 1. Ultrasond travel time (unit: $\mu \mathrm{s}$ ) in well-compacted CFST specimens measured from experiment

Table 2. Ultrasound travel time (unit: $\mu$ s) in the specimens of NT-1 and -3 measured from experiment $\begin{array}{lll}44.6 & 44.6 & 44.6\end{array}$ 
Table. 4 Ultrasound travel time (unit: $\mu$ s) in the specimens of NT-4, -5 and -6

\begin{tabular}{ccccccccccccc}
\hline Testing points & 1 & 2 & 3 & 4 & 5 & 6 & 7 & 8 & 9 & 10 & 11 & 12 \\
\hline NT-4-H & 46.0 & 46.3 & 46.7 & $\underline{\mathbf{5 0 . 9}}$ & $\underline{\mathbf{5 3 . 8}}$ & $\underline{\mathbf{5 1 . 5}}$ & 47.5 & 45.7 & 46.3 & 45.9 & 46.0 & 46.1 \\
NT-4-G & 46.3 & 46.4 & 47.9 & $\underline{\mathbf{5 1 . 4}}$ & $\underline{\underline{\mathbf{5 5 . 6}}}$ & $\underline{\underline{\mathbf{5 3 . 6}}}$ & 47.0 & 46.2 & 46.1 & 45.6 & 45.8 & 45.7 \\
NT-5-H & 47.0 & 47.7 & 47.4 & $\mathbf{4 6 . 1}$ & $\underline{\mathbf{5 2 . 8}}$ & $\underline{\mathbf{5 6 . 5}}$ & $\underline{\mathbf{5 8 . 3}}$ & $\underline{\mathbf{5 7 . 0}}$ & $\underline{\mathbf{6 8 . 9}}$ & 47.2 & 45.2 & 45.0 \\
NT-5-G & 44.9 & 45.6 & 45.8 & 45.8 & $\underline{\mathbf{5 6 . 2}}$ & $\underline{\mathbf{5 7 . 3}}$ & $\underline{\mathbf{5 8 . 7}}$ & $\underline{\mathbf{5 6 . 5}}$ & $\underline{\mathbf{6 7 . 9}}$ & 46.7 & 44.9 & 44.5 \\
NT-6-H & $\underline{\mathbf{5 4 . 8}}$ & $\underline{\mathbf{5 6 . 0}}$ & 46.0 & 45.9 & $\mathbf{4 5 . 6}$ & $\mathbf{4 5 . 5}$ & $\underline{\mathbf{4 8 . 0}}$ & $\underline{\mathbf{5 3 . 7}}$ & $\underline{\mathbf{5 7 . 8}}$ & $\underline{\mathbf{5 8 . 7}}$ & $\underline{\mathbf{6 1 . 2}}$ & $\underline{\mathbf{5 8 . 9}}$ \\
NT-6-G & $\underline{\mathbf{5 5 . 0}}$ & $\underline{\mathbf{5 2 . 3}}$ & 46.6 & 46.5 & 46.4 & 46.7 & $\underline{\mathbf{5 0 . 8}}$ & $\underline{\mathbf{5 5 . 9}}$ & $\underline{\mathbf{5 7 . 6}}$ & $\underline{\mathbf{6 0 . 1}}$ & $\underline{\mathbf{6 1 . 0}}$ & $\underline{\underline{\mathbf{6 0 . 9}}}$ \\
\hline
\end{tabular}

657

658

659

660

\begin{tabular}{|c|c|c|c|c|c|c|c|c|c|c|c|c|}
\hline Testing points & 1 & 2 & 3 & 4 & 5 & 6 & 7 & 8 & 9 & 10 & 11 & 12 \\
\hline NT-4-H & 44.6 & 44.6 & 44.6 & 47.8 & 51.7 & 47.8 & 44.6 & 44.6 & 44.6 & 44.6 & 44.6 & 44.6 \\
\hline & 4.6 & 44.6 & 44.6 & 17.8 & 51.7 & 7.8 & 44.6 & 44.6 & 44.6 & 44.6 & 44.6 & 44.6 \\
\hline & 44.6 & 44.6 & 44.6 & & 49.0 & 2.8 & 55.8 & 52.8 & $\underline{49.0}$ & 44.6 & 44.6 & 44.6 \\
\hline & 44.6 & 44.6 & 44.6 & 44.6 & $\underline{49.0}$ & 2.8 & $\underline{55.8}$ & $\underline{52.8}$ & $\underline{49.0}$ & 44.6 & 44.6 & 44.6 \\
\hline & $\underline{52.5}$ & 48.7 & 44.6 & 44.6 & 44.6 & & 44.6 & 48.7 & $\underline{52.5}$ & $\underline{55.7}$ & $\underline{58.1}$ & $\underline{55.7}$ \\
\hline NT-6-G & $\overline{52.5}$ & $\overline{48.7}$ & 44.6 & 44.6 & 44.6 & 44.6 & 44.6 & $\overline{48.7}$ & $\overline{52.5}$ & $\overline{55.7}$ & $\overline{58.1}$ & 55.7 \\
\hline
\end{tabular}

661

662

663

\begin{tabular}{cccc}
\hline $\begin{array}{c}v_{s} \\
(\mathrm{~m} / \mathrm{s})\end{array}$ & $\begin{array}{c}v_{c} \\
(\mathrm{~m} / \mathrm{s})\end{array}$ & $\begin{array}{c}\text { Minimum of central angle } \\
\left({ }^{\circ}\right)\end{array}$ & $\begin{array}{c}\text { Maximum of central angle } \\
\left({ }^{\circ}\right)\end{array}$ \\
\hline \multirow{6}{*}{5620} & 4000 & 12 & 22 \\
& 4200 & 12 & 45 \\
& 4400 & 12 & 68 \\
& 4600 & 12 & 91 \\
& 4800 & 12 & 110 \\
& 5000 & 12 & 130 \\
& 5200 & 12 & 150 \\
& 5400 & 12 & 172 \\
\hline
\end{tabular}

